\title{
EFFICACY OF ORAL REFRESHER STRIP CONTAINING FIVE TYPES OF ESSENTIAL OILS ON ORAL MALODOR
}

\section{FIESTA ELLYZHA ${ }^{1}$, ANTON RAHARDJO ${ }^{2 *}$, SRY ANGKY SOEKANTO ${ }^{3}$}

${ }^{1}$ Department of Dentistry, Faculty of Dentistry, Universitas Indonesia, Depok, Indonesia. ${ }^{2}$ Department of Preventive and Public Health Dentistry Faculty of Dentistry, Universitas Indonesia, Jakarta, Indonesia. ${ }^{3}$ Department of Oral Biology, Faculty of Dentistry, Universitas Indonesia, Jakarta, Indonesia. Email: antonrahardjo@gmail.com

Received: 21 April 2017, Revised and Accepted: 13 July 2017

\section{ABSTRACT}

Objective: The aim of this study was to determine the efficacy of an oral freshener strip containing five types of essential oils on oral malodor

Methods: This study was a clinical trial using cross-over, randomized-double-blind, and placebo-controlled trial design with 15 male participants. The measured parameters were tongue coating, organoleptic score, and volatile sulfur compounds (VSCs) score.

Results: After the essential oil strip dissolved in the oral cavity, there was no significant reduction in tongue coating, but there was significant reduction $(\mathrm{p}<0.05)$ in the organoleptic score until 60 minutes after dissolved and VSCs until 30 minutes after dissolved.

Conclusion: Essential oil strips are more effective than placebo strips in reducing oral malodor.

Keywords: Oral freshener strips, Oral malodor, Essential oils, Volatile sulfur compounds.

(C) 2017 The Authors. Published by Innovare Academic Sciences Pvt Ltd. This is an open access article under the CC BY license (http://creativecommons. org/licenses/by/4. 0/) DOI: http://dx.doi.org/10.22159/ajpcr.2017.v10s5.23091

\section{INTRODUCTION}

Oral malodor is the most complained about problem from patients visiting the dentist after dental caries and periodontal disease [1]. Oral malodor is an unpleasant or smelly breath emanating from the mouth that is caused by oral or non-oral factors. Oral malodor is generally related to an oral cause $[2,3]$.

Physiological oral malodor is caused by a bacterial putrefactive process occurring within the oral cavity that converts protein into amino acid so that volatile sulfur compounds (VSCs) causing oral malodor are produced [4]. Anaerob Gram negative bacteria are most likely the main causes of oral malodor. They are Porphyromonas gingivalis, Fusobacterium nucleatum, Prevotella intermedia, and Treponema denticola [5]. These can be triggered by bad oral hygiene, reduced oral activity when sleeping, and xerostomia [6]

Approaches that can be taken to eliminate physiological oral malodor are conducting proper oral hygiene measures such as tooth brushing, interdental flossing, and scraping the dorsum of the tongue to clean the tongue coating $[7,8]$. Tongue coating is a white layer lying on the dorsum of the tongue where anaerobic bacteria that can produce VSCs are embedded $[7,9]$. Antibacterial mouthwash is widely used as an additional therapy to treat oral malodor. However, some mouthwashes have additional effects such as bitterness, burning sensation, and tooth staining [3].

Besides antibacterial mouthwash, nowadays, there is another therapy believed to overcome oral malodor - the oral refresher strip. This additional therapy is far more practical and can be used anytime and anywhere. One type of oral freshener strip contains antibacterial essential oils (EOS) such as thymol, eucalyptol, peppermint oil, menthol, and methyl salicylate. These EOS have antibacterial properties that break bacterial cell membrane, interfere with enzymatic processes, and disrupt bacterial lipopolysaccharide [10].

Many researchers who tested EOS in the form of mouthwash proved that these ingredients significantly reduce VSCs producing bacteria that cause oral malodor [10]. However, the efficacy of EOS in the form of oral refresher strips has not been tested. Therefore, this study aimed to compare the effectiveness of a placebo strip (PS) with an oral refresher strip containing five types of EOS: Thymol, eucalyptol, peppermint oil, menthol, and methyl salicylate in reducing physiological oral malodor parameters.

\section{METHODS}

This study was a clinical trial using a cross-over, randomized-doubleblind, and placebo-controlled trial. All protocols used in this research have been reviewed and approved by the Research Ethics Committees of the Faculty of Dentistry at the University of Indonesia, number 180/Ethical Clearance/FKGUI/XI/2012. Researchers selected male participants to avoid the influence of the menstrual cycle on VSCs gas production [11]. Fifteen male volunteers were selected according to inclusion and exclusion criteria to participate in this study.

\section{Inclusion criteria}

Adult males (18-55-year-old) were selected with good general and oral health; non-smoking; had no respiratory problems, such as tonsillitis, bronchitis, bronchiectasis, pulmonary infection, or tumor; had no digestive problems, such as esophageal reflux and pyloric stenosis; had no systemic diseases, such as diabetes mellitus, kidney failure, hepatic disease, and trimethylaminuria; did not use orthodontic or prosthetic appliances; and were willing to participate in all research activities by signing the written informed consent.

\section{Exclusion criteria}

Exclusion criteria had abnormality within the oral cavity, such as active dental caries, periodontal disease, stomatitis aphtosa recurent were undergoing antibiotic therapy the preceding 4 weeks; had taken a xerostomia inducing drug, such as antihistamine, antidepressant, antihypertensive, narcotic were allergic to some of the tested materials.

Each participant received two different treatments 2 weeks in a row. Participants consumed two types of oral refresher strip - A PS and a 
strip containing five types of EOS - On different randomized weeks. Oral malodor parameters were evaluated in this research: Tongue coating score, organoleptic score, and VSCs score.

The tongue coating score was measured both before the strip was consumed and after the strip dissolved by instructing the participants to open their mouth and sticking their tongue out. The area that was covered by the tongue coating was observed then classified by measuring the covered tongue area and receiving a score ranging from 0 to 3 .

The organoleptic score and VSCs were measured before the strip was consumed, immediately after the strip dissolved, 30 minutes after the strip dissolved, and 60 minutes after the strip dissolved. The organoleptic score was measured by asking the participants to close their mouth and breathe through the nose for 30 seconds. Observers and participants were positioned face-to-face with a piece of cardboard that had been hollowed in between. Participants were asked to breathe through a straw slowly. Participants' breath was smelled by the observer. Oral malodor intensity from that breath was scored from 0 to 5 .

VSCs measurements were made using OralChroma. Participants were instructed to close their mouth and breathe through the nose for 30 seconds. A plastic disposable syringe with $2.5 \mathrm{ml}$ volume was inserted in the mouth through the lips and teeth left occluded. The syringe plunger was pulled slowly, then pushed, and pulled for a second time followed by withdrawing it from the mouth. After that, a needle was mounted on the syringe, $1 \mathrm{ml}$ of gas sample from the syringe was injected into the injection port on the OralChroma, and then, the calculation started automatically.

On the day, the experiment was held, participants were requested not to do oral activity such as eating, drinking, and brushing at least $4 \mathrm{hrs}$ before the initial measurement until all the measurements were taken. This was consistent with the Acceptance Program Guideline released by the American Dental Association at the Council on Scientific Affairs 2003. According to Tonzetich, the effect from oral activity will disappear in $3 \mathrm{hrs}$ [12]. Following the initial measurement of oral malodor parameters, participants were requested to consume one type of oral refresher strip (randomized as either EOS or PS). Participants were instructed to consume two of the same strips at once by placing the strips on the tongue and letting them dissolve in saliva. Right after the strips dissolved, the tongue coating score, organoleptic score, and VSCs score were measured. Participants were given a washout period of 1 week to eliminate the residual effects. After the washout period, participants returned to the study and were given two strips of the opposite type of strip (either EOS or PS) to consume, and oral malodor measurements using the same method were performed again. Statistical analysis in this research used the Friedman test and Wilcoxon signed-rank test.

\section{RESULTS}

\section{Tongue coating}

The Wilcoxon signed-rank test showed no statistically significant differences with $\mathrm{p}=0.655(\mathrm{p}>0.05)$ among EOS and PS groups regarding the tongue coating score. The mean tongue coating score was not reduced after PS dissolved inside the mouth and there was a $6.5 \%$ reduction of the mean tongue coating score after EOS dissolved inside the mouth (Fig. 1). Yet, after tested with the Wilcoxon non-parametric test, these reductions were not statistically significant, with $\mathrm{p}=0.157$ $(p>0.05)$.

\section{Organoleptic score}

There was a difference in mean organoleptic score between EOS and PS groups before treatments were given, that is, $3.67 \pm 0.49$ and $3.40 \pm 0.63$, respectively (Fig. 2). Reduction of organoleptic score right after, 30 minutes after, and 60 minutes after EOS dissolved inside the mouth are $81.74 \%, 63.76 \%$, and $58.31 \%$, respectively.
After testing using the non-parametric Wilcoxon test, that difference was not statistically significant, with $p=0.206(p>0.05)$. Furthermore, the Friedman test was employed to see the score changes for each group. There were reductions in organoleptic scores on the PS group, but those reductions were not statistically significant, with $\mathrm{p}=0.169$ ( $p>0.05)$. While there were also reduction organoleptic scores on the EOS group, the Friedman test showed $\mathrm{p}<0.01$ for the reduction of organoleptic scores on the EOS group. Therefore, the reductions of organoleptic scores on the EOS group were statistically significant. Hence, post hoc analysis needed to be taken using the Friedman test to see which intermeasurements of the EOS group differed significantly. According to post hoc analytic using the Friedman test, there were statistically significant differences between organoleptic score before and right after strips dissolved, before and 30 minutes after strips dissolved, and before and 60 minutes after strips dissolved in the EOS group. It was concluded that there were statistically significant differences in the EOS group until 60 minutes after treatment.

\section{VSCs}

There were increases of VSCs scores after PS dissolved inside the mouth followed by reductions and there was reduction of the mean VSCs score after EOS dissolved inside the mouth (Fig. 3).

According to these results, it can be concluded that the data distribution are not normal and the Friedman non-parametric test needed to be taken

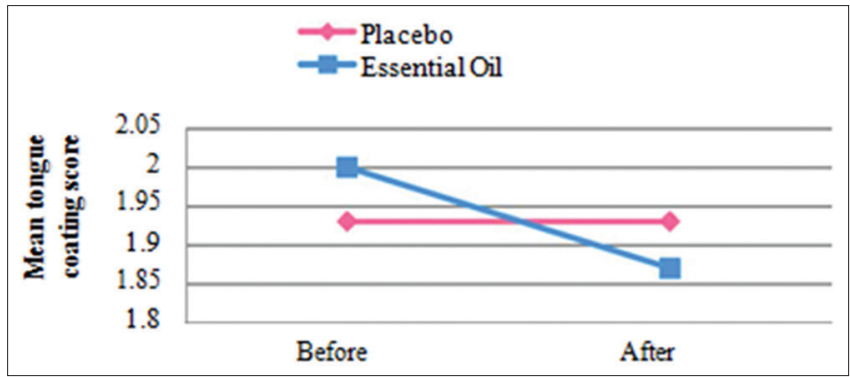

Fig. 1: Mean tongue coating score changes

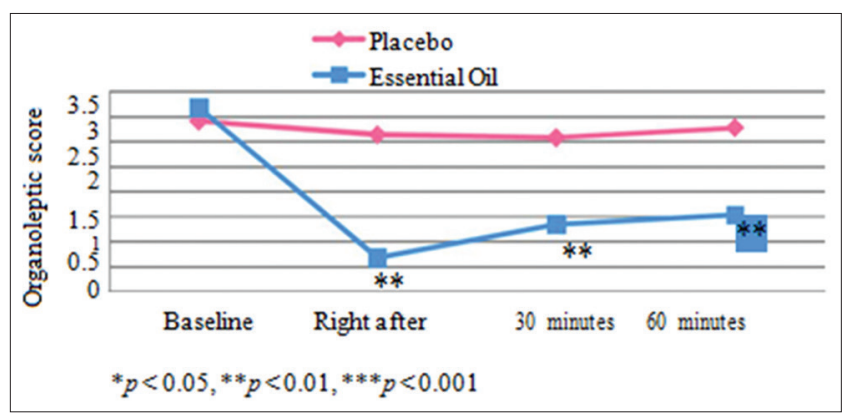

Fig. 2: Mean organoleptic score changes

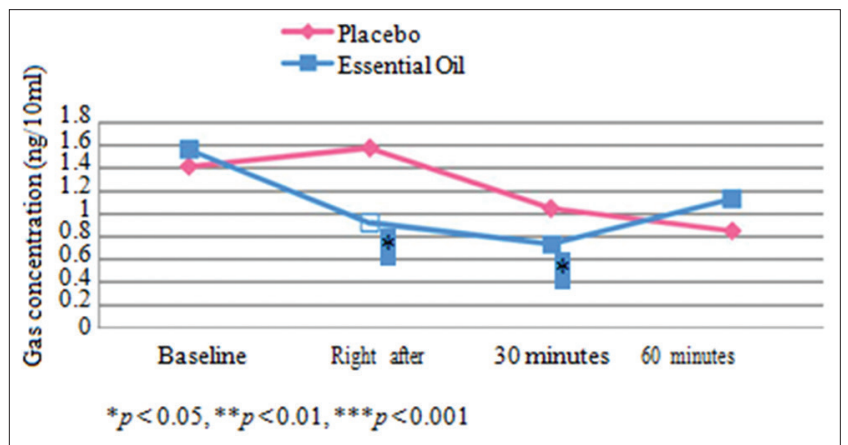

Fig. 3: Mean total volatile sulfur compounds score changes 
to determine whether those reductions were statistically significant. The Wilcoxon test revealed that the difference of mean VSCs score on the PS group was not statistically significant, with $\mathrm{p}=0.320$; meanwhile, the difference of mean VSCs score on the EOS group was statistically significant, with $\mathrm{p}=0.016$. To know which intermeasurement of EOS group was significantly different, it was mandatory to take post hoc analytic using the Friedman test.

In accordance with the post hoc analytic using the Friedman test, $\mathrm{p}=0.007$ was found for before and right after EOS dissolved, and $\mathrm{p}=0.005$ for before and 30 minutes after EOS dissolved. It could be concluded that there were statistically significant differences among before, right after, and 30 minutes after EOS dissolved. VSCs reductions for the previous measurements were $41.15 \%, 53.25 \%$, and $27.5 \%$, respectively.

Among the three types of VSCs gas, there were significant reductions of $\mathrm{H}_{2} \mathrm{~S}$ and $\mathrm{CH}_{3} \mathrm{SH}$ until 30 minutes after EOS dissolved inside the mouth, while there was no significant reduction of $\left(\mathrm{CH}_{3}\right)_{2} \mathrm{~S}$ after EOS dissolved (Figs. 4-6)

\section{DISCUSSION}

This study compared the efficacy of EOS to reduce oral malodor parameters after consumption of EOS and PS. A tongue coating score

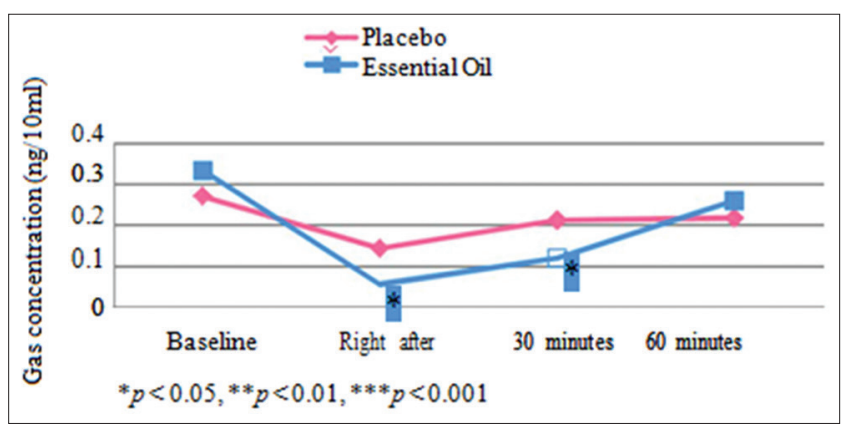

Fig. 4: Mean $\mathrm{H}_{2} \mathrm{~S}$ score changes

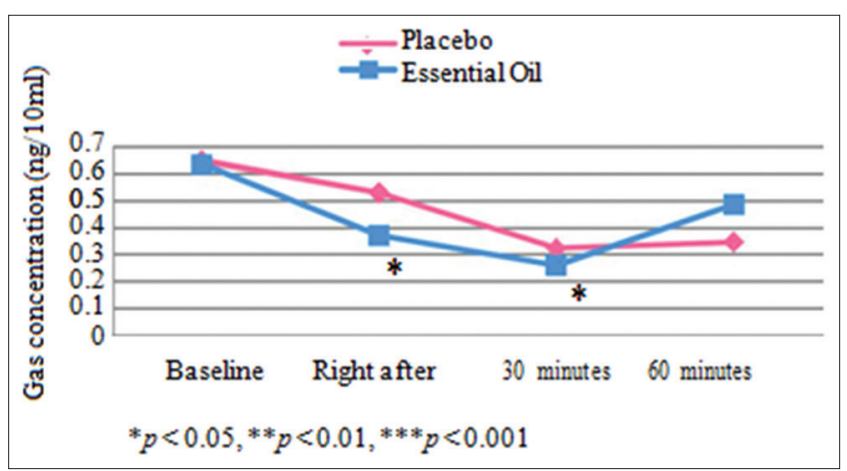

Fig. 5: Mean $\mathrm{CH}_{3} \mathrm{SH}$ score changes

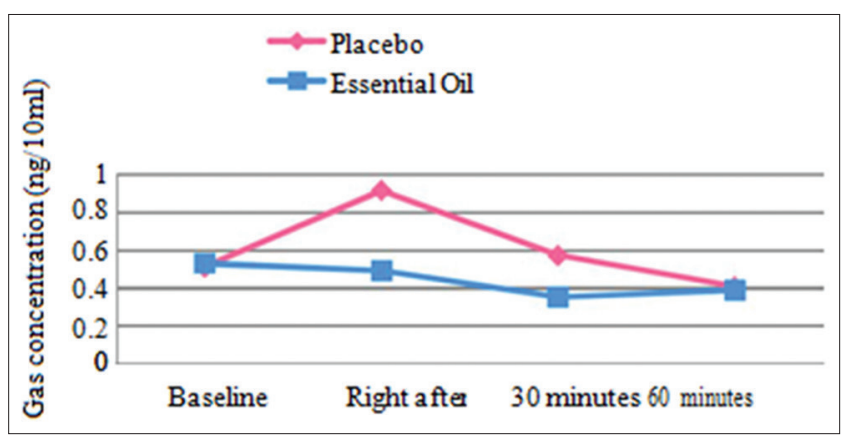

Fig. 6: Mean $\left(\mathrm{CH}_{3}\right)_{2} \mathrm{~S}$ score changes was recorded before strip consumption and after strip dissolved. Organoleptic and VSCs scores were recorded before strip consumption, 30 minutes after strip dissolved, and 60 minutes after strip dissolved. Researchers chose those periods to evaluate how long the effectiveness of EOS could withstand on reducing organoleptic score and VSCs. Power of estimation that was used was $95 \%$ with the type of error that could occur being type 1 where the right null hypothesis was rejected. The tongue coating score was measured visually with a score ranging from 0 to 3. Statistical analysis showed that there was no significant reduction of mean tongue coating score after PS dissolved, but there was $6.5 \%$ reduction in the mean tongue coating score after EOS dissolved, however, this reduction was not significant. Therefore, EOS was not effective in reducing the tongue coating score. This was because some previous researchers revealed that tongue coating is difficult to eliminate even with mechanical approaches [8].

The tongue coating visual measurement method is the fastest and most practical one, but the shortcoming of this method is the visual variability from the observer and ambiguity due to tongue morphological differences [13]. Previous study by Thaweboon (2011) signified that rinsing with mouthwash containing some EOS can have a significant effect on VSC-producing bacteria on the tongue [14]. Microbiological assay of dorsal tongue's bacteria needs to be carried out to attest to EOS efficacy in a more objective way.

After PS dissolved, there was no significant organoleptic score reduction, but there was significant organoleptic score reduction after EOS dissolved. The reduction of organoleptic scores right after, 30 minutes after, and 60 minutes after EOS dissolved was 81.74\%, $63.76 \%$, and $58.31 \%$, respectively. It was concluded that EOS was effective in reducing organoleptic score until 60 minutes after strip dissolved. The gold standard in measuring oral malodor is using the organoleptic method. Nevertheless, this method has several shortcomings: It tends to be subjective and is influenced by many factors from the examiner such as fatigue [15]. To minimize bias, the examiner must be trained and calibrated before taking measurements. Some literature says that this organoleptic method must be conducted by more than two calibrated examiners [16]. Other literature suggests that organoleptic measurement is better carried out by one skilled examiner to avoid interexaminer variation. In this case, organoleptic measurement performed by one examiner to avoid interexaminer bias is recommended by Brunner [17].

Besides tongue coating and organoleptic score measurement, researchers also measured the individual score of VSCs gas using OralChroma. Mean VSCs of all participants before the treatment was $1.41 \pm 0.97 \mathrm{ng} / 10 \mathrm{ml}$ for PS group, and $1.56 \pm 1.25 \mathrm{ng} / 10 \mathrm{ml}$ for EOS group. Participants' highest VSCs score was for $\mathrm{CH}_{3} \mathrm{SH}$, which was $0.65 \pm 0.58 \mathrm{ng} / 10 \mathrm{ml}$ for PS group and $0.63 \pm 0.57 \mathrm{ng} / 10 \mathrm{ml}$ for EOS group. Mean $\left(\mathrm{CH}_{3}\right)_{2} \mathrm{~S}$ score was $0.51 \pm 0.62 \mathrm{ng} / 10 \mathrm{ml}$ for PS group and $0.53 \pm 0.53 \mathrm{ng} / 10 \mathrm{ml}$ for EOS group. $\mathrm{H}_{2} \mathrm{~S}$ had the lowest score, which was $0.27 \pm 0.39 \mathrm{ng} / 10 \mathrm{ml}$ for PS group and $0.33 \pm 0.34 \mathrm{ng} / 10 \mathrm{ml}$ for EOS group. This showed that oral malodor may arise if there is no oral activity done in $4 \mathrm{hrs}$ [12]. That condition was proved by the mean of $\mathrm{CH}_{3} \mathrm{SH}>0.5 \mathrm{ng} / 10 \mathrm{ml}$ and the mean of $\left(\mathrm{CH}_{3}\right)_{2} \mathrm{~S}>0.2 \mathrm{ng} / 10 \mathrm{ml}$, which is the threshold by which an odor can be smelled by the human sense of smell, according to Tonzetich criteria.

VSCs scores were reduced significantly right after EOS dissolved until 30 minutes after EOS dissolved, compared to the score before treatment was given. VSCs scores reduction right after, 30 minutes after, and 60 minutes after treatment were $41.15 \%, 53.25 \%$, and $27.5 \%$, respectively. There was no significant change of VSCs score after PS dissolved inside the mouth. This showed that EOS was more effective to reduce VSCs score until 30 minutes compared with PS. A study by Bercy found that mouthwash containing EOS can kill anaerobic VSCs producing bacteria by breaking the bacterial cell membrane and inhibiting their enzymatic activity [10]. That research implied that EOS could reduce VSCs score. 
Significant reduction of VSCs after EOS dissolved inside the mouth occurred to $\mathrm{H}_{2} \mathrm{~S}$ and $\mathrm{CH}_{3} \mathrm{SH}$ until 30 minutes after dissolved, while reduction of $\left(\mathrm{CH}_{3}\right)_{2} \mathrm{~S}$ was not statistically significant. Based on the literature, $\mathrm{H}_{2} \mathrm{~S}$ and $\mathrm{CH}_{3} \mathrm{SH}$ play a greater role in producing oral malodor than $\left(\mathrm{CH}_{3}\right)_{2} \mathrm{~S}$ [18]. Reduction of mean $\mathrm{H}_{2} \mathrm{~S}$ right after and 30 minutes after EOS dissolved were $83.44 \%$ and $64.46 \%$, respectively. Reduction of mean $\mathrm{CH}_{3} \mathrm{SH}$ right after and 30 minutes after EOS dissolved were $42.85 \%$ and $59.10 \%$, respectively. The biggest reduction occurred on $\mathrm{H}_{2} \mathrm{~S}$. Therefore, EOS has a better ability to eliminate physiological oral malodor because $\mathrm{H}_{2} \mathrm{~S}$ findings were more common in physiological oral malodor patients. Meanwhile, $\mathrm{CH}_{3} \mathrm{SH}$ findings were more common in oral malodor patients with periodontal disease [19]. However, $\mathrm{CH}_{3} \mathrm{SH}$ was also found in patients with healthy periodontal tissue with a lower concentration than in patients with periodontal disease because bacteria on the tongue surface can produce those gases [8].

There were differences in effectiveness of EOS in organoleptic score and VSCs score. On organoleptic measurement, EOS was effective in reducing oral malodor intensity until 60 minutes after the strip dissolved rather than score before treatment was performed. Meanwhile, significantly reduced VSCs score recorded only until 30 minutes after EOS dissolved rather than score before treatment was performed. Researchers attempted to explain this phenomenon with two possible reasons. First, organoleptic measurement cannot differentiate the types of gases contained in mouth air, while OralChroma can detect three types of VSCs gases: $\mathrm{H}_{2} \mathrm{~S}, \mathrm{CH}_{3} \mathrm{SH}$, and $\left(\mathrm{CH}_{3}\right)_{2} \mathrm{~S}$ [15]. Based on literature, gases that contribute to oral malodor were not only VSCs but some other gases such as cadaverine, putrescine, indole, and skatole, which contribute a little (Tonzetich, 1978) [20]. Researchers presumed that EOS could reduce some other gases except VSCs. Second, peppermint oil contained in EOS caused a masking effect against VSCs [21]. That is why in organoleptic measurement, the masking effect made VSCs unable to be sensed.

This study revealed that EOS was not effective in reducing tongue coating but was effective in reducing organoleptic and VSCs scores. EOS was considered effective in reducing physiological oral malodor because the most reliable methods to test an oral malodor remover product are organoleptic and VSCs measurements $[3,16]$. Researchers added a tongue coating parameter to give additional evidence because tongue coating is highly related to oral malodor [7]. If the strip was effective in reducing tongue coating score, the strip could potentially reduce oral malodor for a longer time. Strip inability to reduce tongue coating was in accordance with its ability to reduce VSCs that last for only 30 minutes. This fact showed that the strip eliminated oral malodor only temporarily. The masking effect from the strip was expected to be strong because it can reduce organoleptic score in all measurement periods. That is why an EOS strip can be a promising alternative therapy to reduce physiological oral malodor. However, the strip cannot substitute for mechanical oral hygiene procedures like brushing since traditional oral hygiene methods still provide the most reliable way to eliminate physiological oral malodor $[7,8]$.

The weakness of this study is the limited research period so that the long-term effect of an oral refreshed strip containing active EOS, such as thymol, eucalyptol, peppermint oil, menthol, and methyl salicylate, could not be explored. Moreover, the limited number of samples (15) made the data not representative. However, using a cross-over trial design, researchers could get up to 30 data points, which lessened the shortcoming of the limited samples. Because of this, further study with the use of more samples, durations, and more frequent essential oil strip administration is needed to get more representable data and so that the long-term effect of strip consumption can be learned.

\section{CONCLUSION}

An oral refresher strip containing five types of EOS is more effective to reduce physiological oral malodor than a PS. There were significant reductions of VSCs score right after refresher strip containing EOS dissolved inside the mouth and 30 minutes after strip dissolved. There was no significant reduction of tongue coating score and organoleptic score after refresher strip containing EOS and PS dissolved. $\mathrm{H}_{2} \mathrm{~S}$ had the greatest reduction among other VSCs gases right after refresher strip containing EOS dissolved, and 30 minutes after EOS dissolved inside the mouth.

\section{REFERENCES}

1. Loesche WJ, Kazor C. Microbiology and treatment of halitosis. Periodontol 2000 2002;28:256-79.

2. Panicker K, Devi R, Honibald EN, Prasad AK. Oral malodor: A review. J Indian Acad Dent Spec Res 2015;2:49-54

3. van den Broek AM, Feenstra L, de Baat C. A review of the current literature on aetiology and measurement methods of halitosis. J Dent 2007;35(8):627-35.

4. Krespi YP, Shrime MG, Kacker A. The relationship between oral malodor and volatile sulfur compound-producing bacteria. Otolaryngol Head Neck Surg 2006;135(5):671-6.

5. Cortelli JR, Barbosa MD, Westphal MA. Halitosis: A review of associated factors and therapeutic approach. Braz Oral Res 2008;22 Suppl 1:44-54.

6. Pintauli S. The problem of halitosis and its management. Dentika Dent J 2008;13(1):74-9.

7. Hughes FJ, McNab R. Oral malodour - A review. Arch Oral Biol 2008;53 Suppl 1:S1-7.

8. Bollen CM, Beikler T. Halitosis: The multidisciplinary approach. Int J Oral Sci 2012;4(2):55-63.

9. Tanwir F, Momin IA. Halitosis. Pak Oral Dent J 2001;31(2):304-8.

10. Bercy P, Lasserre J. Susceptibility to various oral antiseptics of Porphyromonas gingivalis W83 within a biofilm. Adv Ther 2007;24(6):1181-91.

11. Calil CM, Lima PO, Bernardes CF, Groppo FC, Bado F, Marcondes FK. Influence of gender and menstrual cycle on volatile sulphur compounds production. Arch Oral Biol 2008;53(12):1107-12.

12. Lodhia P, Yaegaki K, Khakbaznejad A, Imai T, Sato T, Tanaka T, et al. Effect of green tea on volatile sulfur compounds in mouth air. J Nutr Sci Vitaminol (Tokyo) 2008;54(1):89-94.

13. Kim J, Jung Y, Park K, Park JW. A digital tongue imaging system for tongue coating evaluation in patients with oral malodour. Oral Dis 2009;15(8):565-9

14. Thaweboon S, Thaweboon B. Effect of an essential oil-containing mouth rinse on VSC-producing bacteria on the tongue. Southeast Asian J Trop Med Public Health 2011;42(2):456-62.

15. Yaegaki K, Coil JM. Examination, classification, and treatment of halitosis; Clinical perspectives. J Can Dent Assoc 2000;66(5):257-61.

16. Greenman J, El-Maaytah M, Duffield J, Spencer P, Rosenberg M, Corry $\mathrm{D}$, et al. Assessing the relationship between concentrations of malodor compounds and odor scores from judges. J Am Dent Assoc 2005;136(6):749-57.

17. Brunner F, Kurmann M, Filippi A. The correlation of organoleptic and instrumental halitosis measurements. Schweiz Monatsschr Zahnmed 2010;120(5):402-8.

18. Nakano Y, Yoshimura M, Koga T. Correlation between oral malodor and periodontal bacteria. Microbes Infect 2002;4(6):679-83.

19. Murata T, Rahardjo A, Fujiyama Y, Yamaga T, Hanada M, Yaegaki K, et al. Development of a compact and simple gas chromatography for oral malodor measurement. J Periodontol 2006;77(7):1142-7.

20. van den Velde S, Quirynen M, van Hee P, van Steenberghe D. Halitosis associated volatiles in breath of healthy subjects. J Chromatogr B Analyt Technol Biomed Life Sci 2007;853(1-2):54-61.

21. Grigoleit HG, Grigoleit P. Pharmacology and preclinical pharmacokinetics of peppermint oil. Phytomedicine 2005;12(8):612-6. 